\title{
Practical Reuse Measurement in ERP Requirements Engineering
}

\author{
Maya Daneva \\ Clearnet Communications, 200 Consilium Place, Suite 1600 \\ Toronto, Ontario M1H 3J3, Canada \\ mdaneva@clearnet.com
}

\begin{abstract}
Component-based, architecture-centric requirements engineering processes have been applied in Enterprise Resource Planning projects for a decade, but have only relatively recently achieved significant recognition in the broader software engineering research community. This sub-area is now entering a new level of maturity by beginning to address the reuse aspects in the ERP RE process. The present article makes a first contribution to applied ERP requirements reuse measurement by incorporating reuse metrics planning as part of the implementation of metrics on an ERP project. Relevant process integration issues are tackled in the context of SAP R/3 implementation projects.
\end{abstract}

\section{Introduction}

Requirements Engineering (RE) forms the major concern in the implementation of any Enterprise Resource Planning (ERP) software package. It is an expensive process referring to the creation, the analysis, the adaptation, and the management of a large number of artifacts, or descriptions. It begins with the identification and the documentation of the company's organizational units, their business processes and data needs and continues throughout the entire implementation cycle in the form of tracking of the life history of any particular requirement and business issue. The better the resulting business requirements are formulated, the faster the progress in subsequent phases, because the necessary decisions concerning the future ERP solution have been taken and agreed upon [19]. To make the RE process more efficient and to assure high quality results, the ERP community has adopted systematic requirements reuse approaches, developed an infrastructure of processes, people and tools for customers to reuse and, most recently, delivered component-based solutions to common business process and data requirements derived from numerous industry-specific business cases. These represent domain-specific frameworks with three major features [13]: an architecture defining the structure of integrated information systems within the business problem domain, a set of business application components engineered to fit the architecture, and a set of tools that assist the consultant in building component-based solutions using the domain knowledge within the architecture. 
Currently, in spite of the increased attention that ERP requirements reuse has attracted, the wide application of reuse process methods and techniques, and the awareness of the benefits they have brought to ERP-clients, very few approaches have emerged to quantitatively measure the results from requirement reuse the customers have achieved [2]. As Pfleeger points out, we 'can not do effective reuse without proper measurement and planning' [14].

The present paper tackles this issue from measurement planning perspective. We present a requirements reuse measurement plan that links the reuse measurement needs to the ERP reuse goals and action items to be taken in the RE process. Our objective is to provide a sound and consistent basis for incorporating reuse metrics planning as part of the implementation of metrics on an ERP project. For the purpose of this research, we place the requirements reuse measurement activities in the context of implementing the SAP R/3 System, a leading product in the ERP software market [19]. However, our approach is generic enough and could easily be applied to any other ERP project implementation. In what follows, we first explain the motivation for our research effort. Then, we discuss the components of our reuse measurement plan and provide some illustrative examples of how measurements are useful. Section 5 generalizes our experience and reports on early lessons learnt and critical success factors. Section 6 concludes the paper and suggests directions for future work.

\section{Motivation}

An ERP requirements reuse measurement process is a systematic method of adopting or adapting standard reuse counting practices in ERP RE, measuring the ERP reuse goals, and indicating reuse levels targeted at the beginning and achieved at the end of each stage of the ERP implementation cycle. The main purpose of this process for ERP customers is to learn about their own business, technological and environment opportunities by learning how much reuse their ERP-supported business processes could practice. The motivation behind the integration of the reuse measurement process in the RE process includes the following:

- Reuse measurement data available early in the project will enable the reuse process to be planned and reuse planning to be done as part of the RE process.

- Reuse metrics will provide a foundation for building and reinforcing partnerships, increasing customers' understanding of the ERP functionality, reprioritizing the business requirements, communicating the value of ERP-reuse.

- Reuse measurement will reduce the probability of errors and accidental omissions in the business process requirements.

- The reuse measurement process will serve as a vehicle for faster resolution of requirements problems and conflict. Metrics data will help focus requirements elicitation and negotiation meetings and resolve architectural problems.

- $\quad$ Reuse data will serve as an input to an effort estimation model. 


\section{Components of a Requirements Reuse Measurement Plan}

The requirements reuse measurement process should be documented in the form of a reuse measurement plan [5]. Its purpose is to establish a reuse measurement practice as part of a larger organizational process, namely, the ERP RE process. Moreover, it represents a communication vehicle to ensure that all the team members agree with the approach as well as serves as the on-going reference model to manage the implementation of reuse metrics. The plan defines the measurement process with exact information on stakeholders involved, measurement frequency, sources of metrics data, counting rules, metrics data interpretation rules, tools support, reports to be produces, and action items that can be taken based on the metrics data (Fig.1.).

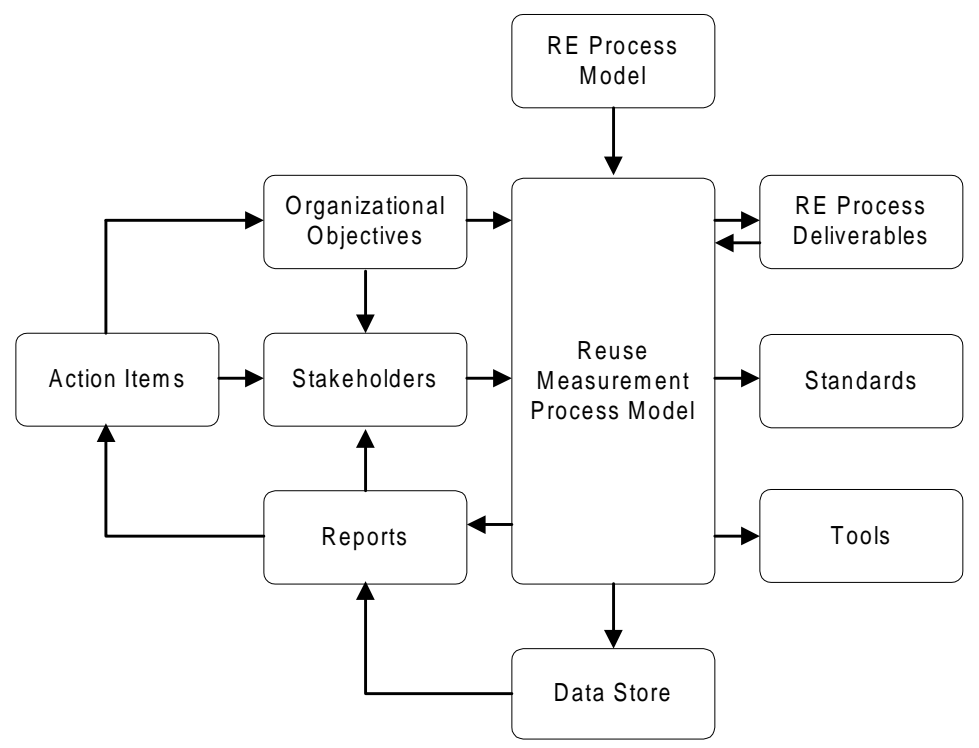

Fig.1. The components of the SAP reuse plan

Stakeholders and their objectives define what is to be achieved by running a reuse measurement process. Next, as per the recommendations provided by software metrics researchers and practitioners [14, 15], a model of the RE process is needed to capture the ERP reuse activities and to understand where measurements fit in. It should provide sufficient knowledge of (i) how to map reuse measures to RE activities, (ii) where and when in the RE process measurements could be taken, and (iii) how measurement activities could be integrated into the larger process. Given this context, a reuse measurement process model is required to specify what to count as requirements reuse, what units of measure to use, and how to count it. Furthermore, tools, data stores and standards for data collection, processing and packaging are to be selected to ensure the quality of the reuse metrics data. Finally, the plan concludes 
with strategies for using the reuse data. These are presented in terms of metrics data reports to be created and action items that can be formulated based on the reported data. The components of our ERP reuse measurement plan are discussed in great detail in the next sections.

\subsection{Understanding Stakeholders and Their Roles}

Adequate and timely consultation of the parties interested in ERP is of great importance to the planning of reuse metrics. It helps us (i) make sure that the definitions of our metrics are based on our SAP team members' goals, (ii) eliminate misunderstandings about how metrics data is expected to be used, and (iii) define relevant procedures for packaging, cataloguing, publishing and reporting reuse metrics data.

To identify the stakeholders, we applied the approach developed by Sharp et all in [17]. Based on early SAP project documentation, we developed stakeholder interaction diagrams that captured three important aspects of our team working environment: relationships between stakeholders, the relationships of each stakeholder to the system, and the priority to be given to each stakeholder's view. The organizational knowledge represented in the diagrams is needed to manage, interpret, balance and process stakeholders' input into the SAP requirements reuse measurement process. It was used to structure the SAP project team members in four groups: (i) business decision makers, who are corporate executives from the steering committee responsible for the optimization, standardization and harmonization of the business processes across multiple locations, and define the concept of ownership over the SAP R/3 system and are most interested in learning about the business benefits from SAP reuse, (ii) business process owners, who are department managers responsible for the project in specific business areas, and contribute the necessary line know-how, design new processes and procedures to be supported by the $\mathrm{R} / 3$ business application components and provide the project with the appropriate authority and resources, (iii) technical decision makers, who are SAP project managers responsible for planning, organizing, coordinating and controlling the implementation project, and (iv) configurators, who are both internal IT team members and external consultants involved in various work packages, e.g. process and data analysts, configuration specialists, ABAP programmers, system testers, documentation specialists. Each stakeholder has its own questions that should be answered by using the metrics data. Business decision makers would like to know:

- What level of standardization could be achieved by reusing ERP software assets?

- What competitive advantages does the team get from ERP reuse?

- What are the implications of reusing ERP processes in a constantly changing business environment?

- How to align business processes across locations so that ERP reuse can yield significant cost reductions and enterprise-wide benefits?

- What implementation strategy fits better with the project?

Business process owners might ask:

- How ERP reuse works with volatile process requirements? 
- How much customization effort is required to implement minor/major changes in the business application components?

- What reuse expectations are realistic?

- What processes have the greatest potential for practicing reuse?

- What activities in our processes prevent us from reusing more?

Technical decision-makers need to know:

- How much effort is required to produce the user and training documentation associated to the customized components?

- How much reuse the team did?

Configurators might ask:

- Are there any rejected requirements that should be re-analyzed because of reuse concerns?

- What implementation alternative fits best?

- Which segments of the requirements are likely to cause difficulties later in the implementation process?

The questions relevant to each group have been documented and attached to the stakeholder interaction diagrams.

\subsection{RE Process Model Outline}

The standard methodology for rapid R/3 implementation, called AcceleratedSAP (ASAP), provides a disciplined reuse-driven, architecture-centric process for coordination, controlling, configuring and managing changes of the R/3 business application components [1,11]. To investigate the ASAP RE process, we modelled it as a spiral (Fig. 2.). Its the radial arms represent the increasing collection of information by three types of activities: (i) requirements elicitation activities which deliver the foundation for the business blueprint and are concerned with finding, communication and validation of facts and rules about the business, (ii) enterprise modelling activities which are concerned with the business processes and data analysis and representation, and (iii) requirements negotiation activities which are concerned with the resolution of business process and data issues, the validation of process and data architectures and the prioritization of the requirements. The ASAP methodology suggests four iterations of the spiral. Level 0 iteration aims at developing a clear picture of the company's organizational structure based on the pre-defined organization units in the $\mathrm{R} / 3$ System. Next, the main objective of level 1 iteration is to define aims and scope for business process standardization based on the R/3 application components. Level 2 iteration aims at deriving company-specific business process architecture based on scenarios from the standard SAP process and data architecture components. Finally, level 3 iteration refers to the specification of data conversion, reporting and interfaces requirements. The major actors in these activities are business process owners who are actively supported by the SAP consultants and the internal SAP process and data architects. Next, the ASAP RE process is supported by the following tools: (i) the ASAP Implementation Assistant [11] which provides reusable questionnaires, project plans, cost estimates, blueprint presentations, blueprint templates, project reports and checklists, as well as manages the documentation base; (ii) the SAP Business 
Engineer, a platform including a wide range of business engineering tools fully integrated into the R/3 System [1]; (iii) enterprise modelling tools (ARIS-Toolset, LiveModel and Visio) which have rich model management capabilities and assist in analyzing, building and validating customer-specific process and data architectures based on the reusable reference process and data models.

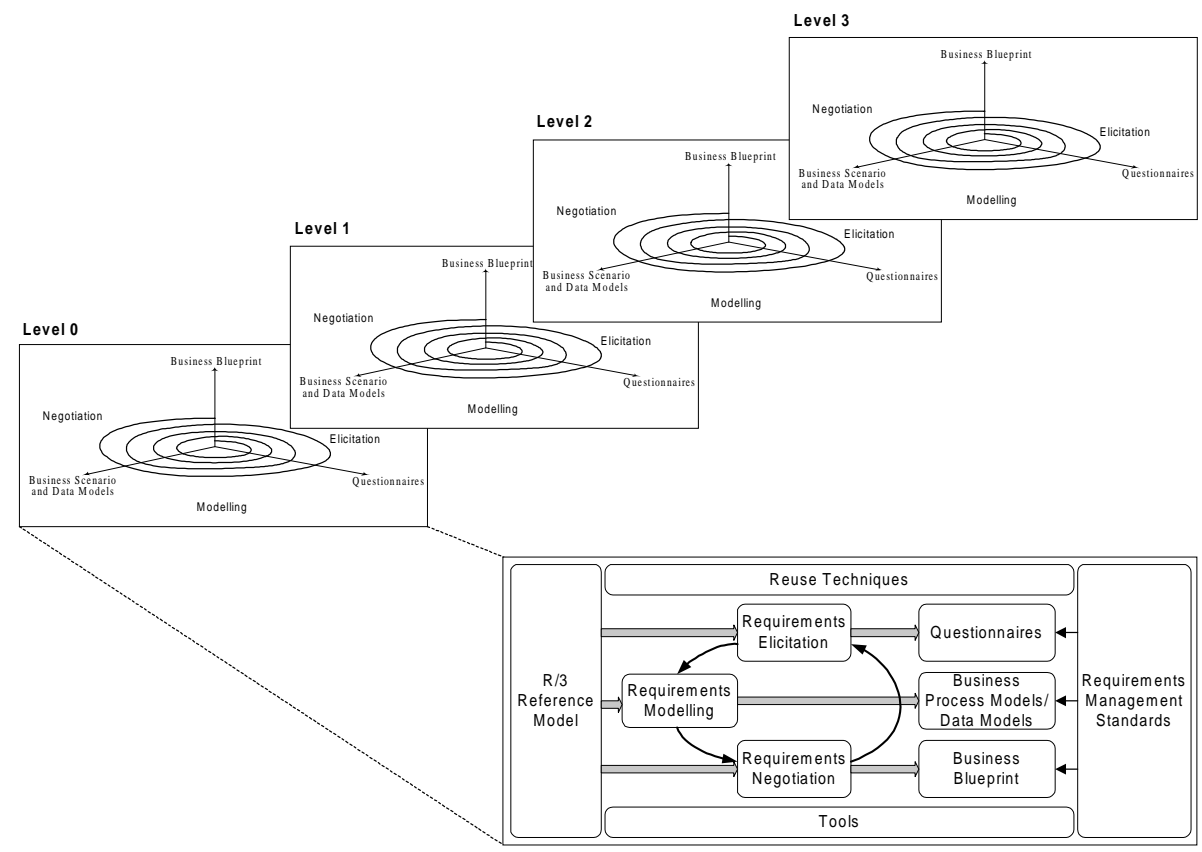

Fig.2. The SAP requirements engineering process

The ASAP RE begins with reuse, ends with reuse and includes reuse in all the tasks in-between. It is based on proven reuse practices and techniques and it ensures that the requirements are correct, consistent, complete, realistic, well prioritized, verifiable, traceable and testable. This is achieved by using the R/3 Reference Model, a comprehensive architectural description of the R/3 System including four views: business process view, function view, data view and organizational view. Specifically, the R/3 Reference Process Models represent integrated and function-spanning collections of business processes that occur often in practice and can be handled to the greatest extend possible automatically if a corporation implements the complete $\mathrm{R} / 3$ System [11]. Instead of building an integrated information system from scratch, with the R/3 Reference Model we build a solution from reusable process and data architectures based on SAP's business experience collected on a large scale. Our analysis indicates that the R/3 Reference Model supports the RE process in multiple ways: (i) in requirements elicitation, it provides a way for process owners and consultants to agree on what the SAP business application components are to do, (ii) in requirements modelling, it serves two separate but related purposes. It helps to 
quickly develop a requirement definition document that shows to the business owners the process flow the solution is expected to support. Beyond that, it can be seen as a design specification document that restates the business specification in terms of $\mathrm{R} / 3$ transactions to be implemented, and (iii) in requirements negotiation, the R/3 Reference Model serves as a validation tool. It makes sure that the solution will meet the owners' needs, it is technically implementable and it is maintainable in future releases.

Reusing architectural components in the RE process is saving both time and money. As the business process requirement analysis is the most expensive consulting service in a business engineering exercise, the reuse of the R/3 Reference Model definitely provides the greatest savings.

\subsection{Process Integration Model}

This section describes exactly how reuse measurement can be integrated with the RE activities and where in the RE process reuse measurement data will be taken (Fig. 3). It involves several assumptions:

- Reuse data are extracted by an SAP process analyst on the basis of two major RE deliverables: business scenario models and business object models;

- reuse metrics data analysis is based on quantitative indicators;

- reuse metrics data is used to support stakeholders' decision during the requirements negotiation and elicitation;

- reuse metrics data is reused at a later stage to support decision making in planning for future releases, upgrades and major enhancements.

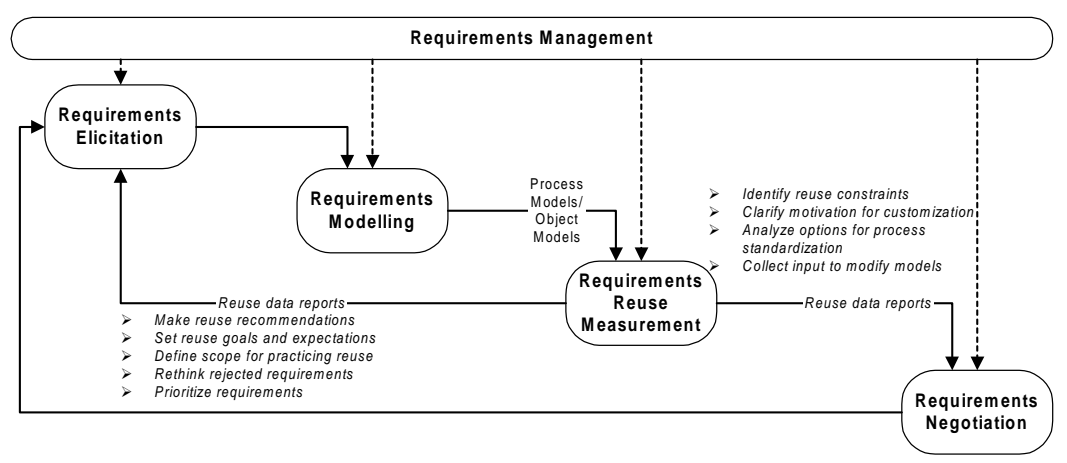

Fig. 3. Integration of requirements reuse measurement in RE

We suggest reuse measurement be applied once the modelling activities of level 2 iteration are completed and the customer-specific process and data architectures are built (Fig.3). Given the reuse metrics data, the SAP process analyst may decide what negotiation / elicitation activities to take place next. The use of the metrics data is discussed in more detail in Section 4. 
Our integration model implies that reuse measurement activities support the RE process in five areas: (i) definition of measurable reuse goals and expectations, (ii) quantitative analysis of process and data architecture reuse prior to solution design; (iii) assessment of the requirements specification, (iv) better understanding of the technical risks early in the ERP implementation cycle, (v) definition of the scope of ERP reuse and how it fits into the business environment.

\subsection{The Measurement Process}

As Pfleeger [14] recommends, we have to choose reuse metrics based on what is visible for the SAP project team in the requirements modelling process of level 2 iteration. Our approach uses the results of our previous research on the derivation of reuse indicators from SAP scenario process models and business object models [2]. It is based on the notion of "reuse percents" [18] suggests a reuse indicator that includes reused requirements as a percentage of total requirements delivered [2]:

$$
\text { SAP_Reuse }=(\text { RR } / \mathrm{TR}) * 100 \%
$$

where $R R$ represents reused requirements, and $T R$ represents total requirements delivered. In this paper, requirement borrowed from the R/3 Reference Model are classified as reusable if it does not require modification. If borrowed requirement does require minor or major enhancement before use, we term it 'customized requirement'.

To build well-defined and valid metrics [7], we selected a consistent and reliable means for structuring and collecting data to make up metrics. A standard functional size measurement methodology, namely Function Point Analysis (FPA) [8] was applied to size the total and the reused requirements in the project. It was chosen because of its appropriateness to the software artifact being measured [8,16] and its proven usage and applicability in software reuse studies $[10,15]$. However, we needed to adapt FPA the SAP requirements. This has been achieved in [3] by defining rules for mapping SAP business process models and data object models to the FPA counting components: we mapped SAP data entities to FPA data types, and SAP process components to FPA transaction types. As a result, the size of a scenario process model is assumed to be a function of the process components included in the model and the data objects defining the data that support the process. The step-by-step procedure for counting Function Points (FP) from scenario process models and business object models is described in [2] and [3] in terms of inputs, outputs and deliverables. Generally, it involves three stages: analysis of the process and data components, assignment of complexity values to the components and calculation of the final FP value.

Based on the analysis of the changes [11] that could be applied to the R/3 Reference Model throughout the reuse-based process modelling exercise, the measurement data collected throughout the FP sizing procedure [3], and the modes of component reuse investigated by Karllson [10], we have defined three levels of requirements reuse [2]:

- Level 3: It refers to process and data components that were reused without any changes. This category of reuse would bring the greatest benefits to the 
SAP customer's organization. Scenarios with higher reuse rate at this level have greater potential of practicing reuse.

- Level 2: It refers to minor enhancements applied to reference processes and data components. A minor enhancement is defined as a change of certain parameter of a business process or a data component that does not result in a change of the process logic. This category of reuse refers to those processes and data components of the R/3 Reference Model that logically match the business requirements but their parameters need to be changed at code level to achieve their business purpose. Level 2 reuse is as desirable as level 3 reuse.

- $\quad$ Level 1: It refers to major enhancements applied to reference processes and data components. A major enhancement is any considerable modification in the definition of a process or a data component that affects the process logic from business user's point of view. This category of reuse refers to those processes and data components that do not match the business requirements and require changes at conceptual level, as well as at design and code level to achieve their business purpose. Level 1 reuse is at least desirable.

In these definitions, the term process (component) refers to the functional units of any SAP scenario process models and the term data component means a data entity, a relationship or an attribute from the data model describing the SAP business data requirements. Furthermore, we introduce a level of new requirements, No_Reuse, to acknowledge the fact that reuse is not practiced at all. It refers to newly introduced processes and data components. This does not mean a reuse category; it just helps us to partition the overall requirements and to get understanding of how much requirements are not covered by the standard scenario processes and business objects.

Given our definition of what to count as reuse and how to count it, we have derived three reuse indicators [2]:

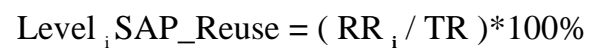

where $\mathrm{i}=\{1,2,3\}, \mathrm{RR}_{\mathrm{i}}$ represents reused requirements at Level $_{i}$, and TR represents total requirements delivered. The indicator

$$
\text { No_Reuse }=(\text { NR / TR }) * 100 \% \text {, }
$$

where NR represents the new requirements, and TR has the above meaning, reports the percentage of requirements that can not be met by the $R / 3$ application package unless some customer-specific extensions are not developed.

Currently, case studies are being carried out to validate empirically our counting model and its application procedure [4]. This exercise is being done on the basis of Jauqet's and Abran's framework [9] for investigating measure validation issues. 


\subsection{Assembling a Toolset for Data Collection}

Reuse measurements are only as good as the data that is collected and analyzed [7]. To assure the quality of the reuse data, at least three tools are needed: (i) a form for recording all the counting details; (ii) a reuse metrics database, and (iii) a process knowledge repository. We extended the FP counting form suggested in [8] by including information needed for calculating the reuse indicators. Based on our FP counting model [3], we devised a counting form usage procedure that indicates at exactly what point each piece of data should be collected. information has been stored and processed in Excel spreadsheet software. Summarized and detailed reports have been extracted from Excel tables. For example, Table 2 shows the size numbers for four SAP business scenarios and Table 3 presents the summarized results from measuring reuse. Since reuse metrics provide knowledge about the business processes, reports on metrics data should be considered as part of the SAP process documentation. It can be stored, packaged, catalogued and published by using a corporate intranet repository as well as standard process modelling tools and the ASAP Implementation Assistant. In this way, data is made available for review and analysis to all interested parties. Users of SAP documentation can easily navigate from scenario process models to functional size and reuse metrics data.

Table 2. Reuse levels for five SAP scenarios

\begin{tabular}{lcccc}
\hline \multicolumn{1}{c}{ Business Scenarios } & Level $_{3}$ & Level $_{2}$ & Level $_{1}$ & No \\
& $F P$ & FP & FP & FP \\
Project-related Engineer-to-Order Production & 141 & 57 & 68 & 128 \\
Procurement of Stock Materials & 74 & 198 & 32 & 127 \\
Procurement of Consumable Materials & 86 & 165 & 0 & 30 \\
External Service Management & 96 & 31 & 14 & 0 \\
\hline
\end{tabular}

Table 3. Reuse levels for five SAP scenarios

\begin{tabular}{lcccc}
\hline \multicolumn{1}{c}{ Business Scenarios } & Level $_{3}$ & Level $_{2}$ & Level $_{1}$ & No \\
& Reuse & Reuse & Reuse & Reuse \\
Project-related Engineer-to-Order Production & $36 \%$ & $15 \%$ & $17 \%$ & $32 \%$ \\
Procurement of Stock Materials & $19 \%$ & $50 \%$ & $8 \%$ & $22 \%$ \\
Procurement of Consumable Materials & $31 \%$ & $59 \%$ & $0 \%$ & $10 \%$ \\
External Service Management & $68 \%$ & $22 \%$ & $10 \%$ & $0 \%$ \\
\hline
\end{tabular}




\subsection{How to Link Reuse Data to Action Items}

Measurements are considered useful if they help stakeholders (i) understand what is happening during the ERP RE process, and (ii) control what is happening on the ERP project [7]. Typically, two types of reuse profiles could be derived from a requirements reuse measurement table (Table 3): scenario-specific profiles which present the levels of reuse pertinent to a given scenario, and level-specific profiles which show how the requirements are reused at a specific level within a project. Business decision-makers can use both types of profiles in at least three ways: (i) multiple reuse profiles of two or more different ERP products (SAP, BAAN, PeopleSoft) can be compared to determine which package best serves the needs of the company and offers the greatest opportunity for reuse; (ii) multiple reuse profiles of different releases (SAP R/3 3.1, 4.0B, 4.5, 4.6 ) of one ERP package could be compared to determine which release brings biggest benefits to the company; (iii) multiple reuse profiles of a single ERP package (e.g. SAP R/3) can build an assessment of the overall level of standardization of the ERP solution in the organization. Reuse profiles of a single ERP package (e.g. SAP R/3) can be used by technical decision-makers to plan and control the reuse levels in the later phases of the ASAP implementation process. Business process owners and configurators can track requirements reuse levels over time to control the changes in overall reuse during the iterations of the RE process.

Furthermore, the specific use of each profile is systematically documented by using a Reuse Data Usage Table. It is built to characterize four aspects of a reuse profile: who needs to read the profile data, what the profile can help us understand, what the profile can help us control and what action items are likely to be taken based on the reuse profile. Tables 4 and Table 5 reports on the current usage of the scenariospecific and level-specific profiles, respectively. (BDM, PO, TDM and C stand for business decision-makers, process owners, technical decision-makers and configurators, respectively.)

\section{Discussion on the Reuse Data Usage}

The examples of Table 2 and 3 show scenarios referring to two SAP components: Project System and Materials Management. The Project-related-Engineer-to-Order scenario describes how to manage projects for building cell sites for a country-wide mobile telephone network. Its Level ${ }_{1}$ Reuse and No_Reuse ratings are relatively high due to significant customization and numerous external interfaces required by the process owners. Next, the scenarios of Procuring Stock Materials and Procuring Consumable Materials refer to the business workflow in purchasing, inventory management, and warehouse operations. They cover the processes of material requirements planning, purchase requisition processing, purchase order processing and goods receipt processing. Furthermore, the External Service Management scenario documents the workflow for service provisioning and handling contractors in cell site construction. This scenario is the one, which practices most Level 3 reuse. 
Table 4. Reuse data usage table for scenario-specific profiles

\begin{tabular}{|c|c|c|c|c|c|}
\hline Usage & BDM & $\mathrm{PO}$ & TDM & $\mathrm{C}$ & Action items \\
\hline $\begin{array}{l}\text { Understand the } \\
\text { customization risk for } \\
\text { upgrade projects. }\end{array}$ & $\mathrm{x}$ & $\mathrm{x}$ & $\mathrm{x}$ & $\mathrm{x}$ & $\begin{array}{l}\text { 1. Assess the difficulty in the migration } \\
\text { of processes with low reuse rates. } \\
\text { 2. Reengineer the business requirements. } \\
\text { 3. Budget and plan resources for extra } \\
\text { gap analysis for the processes with low } \\
\text { reuse rates. }\end{array}$ \\
\hline $\begin{array}{l}\text { Understand how } \\
\text { much reuse the team } \\
\text { did. }\end{array}$ & & $\mathrm{x}$ & $\mathrm{x}$ & $\mathrm{x}$ & $\begin{array}{l}\text { 1. Set reuse expectations for later stages. } \\
\text { 2. Define scope for practicing reuse. } \\
\text { 3. Make process reuse recommendations. }\end{array}$ \\
\hline $\begin{array}{l}\text { Understand reuse } \\
\text { constraints / Assess } \\
\text { the level of } \\
\text { standardization. }\end{array}$ & $\mathrm{x}$ & $\mathrm{x}$ & & $\mathrm{x}$ & $\begin{array}{l}\text { 1. Elaborate alternative process flows to } \\
\text { eliminate the need for customization. } \\
\text { 2. Re-assess reuse levels. } \\
\text { 3. Compare processes to select the best } \\
\text { alternative. }\end{array}$ \\
\hline
\end{tabular}

Table 5. Reuse data usage table for level-specific profiles

\begin{tabular}{|c|c|c|c|c|c|}
\hline Usage & BDM & $\mathrm{PO}$ & TDM & $\mathrm{C}$ & Action items \\
\hline $\begin{array}{l}\text { Define focus for } \\
\text { negotiation meetings. }\end{array}$ & & $\mathrm{x}$ & & $\mathrm{x}$ & $\begin{array}{l}\text { 1. Review scenarios on a function-by- } \\
\text { function basis to justify why customi- } \\
\text { zation is necessary. } \\
\text { 2. Structure requirements in three catego- } \\
\text { ries: must-to-have, nice-to-have and } \\
\text { possible-but-could-be-eliminated. }\end{array}$ \\
\hline $\begin{array}{l}\text { Select an } \\
\text { implementation } \\
\text { strategy. }\end{array}$ & $\mathrm{x}$ & $\mathrm{x}$ & $\mathrm{x}$ & & $\begin{array}{l}\text { 3.Consider a step-by-step approach to a } \\
\text { sequenced implementation, if Level } 1 \\
\text { reuse dominates. } \\
\text { 4.Consider a big-bang approach, if Level } \\
3 \text { reuse dominates. }\end{array}$ \\
\hline
\end{tabular}

The scenario-specific data usage table suggests what benefits the reuse measurements bring to those team members who are responsible for planning for reuse and assigning target reuse levels to each scenario to be achieved throughout the R/3 implementation project. Some examples of how these profiles are helpful include the following:

- The data can be used in level 3 requirements elicitation to understand what prevents the team from reusing more. In the Project-related-Engineer-toOrder scenario, the low level of reuse is due to three reasons: (i) the standard $\mathrm{R} / 3$ functionality does not offer sufficient support to the business practices specific to a mobile telecommunication services provider, (ii) numerous external interfaces to legacy systems should be built, and (iii) business processes in cell site construction have not been standardized across the locations: different regional cell site groups wanted to use the SAP syetsm differently. 
- The data are useful in planning for both new implementations and upgrades. In the first case, documentation development risks that were not foreseen may appear for processes with high Level ${ }_{1}$ Reuse or No_Reuse rates. They are likely to need additional resources (e.g. business process owners, internal training specialists, and documentation analysts) to get documented. In case of upgrades, reuse profiles help the team assess the degree of difficulty involved in the migration to the new release. For example, Table 2 suggests that the process of Project-related Engineering-to-Order Production should be migrated with extra caution.

Next, the level-specific usage table is important to requirements negotiation activities. Two illustrative examples refer to the requirements prioritization activities and the implementation strategy selection activities:

- The reuse data help the team decide what to focus the negotiation efforts on. ]. As the process owners get better understanding of the SAP reuse, and recognize the R/3 customization is one of the most risky matters to deal with in the package implementation, they become more conscious to the avoidance of unnecessary adaptation and are willing to re-prioretize the requirements

- The level-specific profiles can help both business and technical decisionmakers determine what SAP implementation strategy fits best with the organizational objectives. If Level 1 reuse dominates and much customization efforts are anticipated, the team is likely to adopt a step-by-step approach to a sequenced implementation of the SAP components. If Level 3 reuse rates are the highest ones, the customization risks are reduced and a big-bang approach to implementing multiple components seems to be reasonable.

\section{Packaging Experiences}

SAP requirements sizing and reuse counting has been practiced in four SAP projects: three new implementations and one upgrade. While applying the process, we collected and documented some facts and observations about the context of reuse measurement. Thus, we obtained a set of experience packages that suggested explanations of how and why the measurement process worked as part of the RE cycle [1]. Each package consists of characteristics of the project context, a logical conclusion about specific aspects of the measurement process and a set of facts and observations that support this conclusion. The conclusions represent either early lessons learnt that tell us what and how worked in the process or critical success factors that suggest why it worked.

A summary of our early lessons learnt is given as follows:

- Requirements reuse measurement helps us understand in both qualitative and quantitative terms the role of the pre-defined process models in ERP RE.

- The measurement process must be focused on defining action items based on the reuse data metrics, not on collecting and reporting data.

- The process leads to consistent traceability information being maintained for all the business processes. 
- It increases the probability of finding poorly prioritized requirements.

- Reuse data is a central record of all the process specific reuse information.

- Reuse data helps to focus the validation process.

- Reuse measurement should not be practiced as a short-term process that would be dropped at the end of the SAP implementation cycle. Measuring requirements reuse should not be considered as one-sided gathering of information. It is neither one-time process knowledge capture, nor a quick fix.

Moreover, 10 critical success factors have been identified:

- Apply a stakeholder identification method to the SAP project organization. This made sure that all important stakeholders have been captured, and yet that irrelevant actors have not been included.

- Use the ASAP standard processes, deliverables and tools. This significantly shortened the time needed to model the RE process and to spot where in this process measurements could be taken, analyzed and used.

- Adopt (if possible) or adapt a standard methodology for sizing the business requirements. FPA proved its usefulness and applicability in ERP RE.

- Integrate the reuse measurement process incrementally. Pilot it by applying it to the business scenarios pertinent to a selected ERP component.

- Consider the metrics data reports as a supplement to the business blueprint. The business process owners should review it as the other deliverables included in the business blueprint.

- Take extra efforts to experiment with the reuse measurement process and to collect and document the series of action items the team members suggest based on the metrics data.

- Understand the role of the reusable components and the reuse techniques in the ERP RE process.

- Maintain a limited number of requirements reuse measurement process documents: it is sufficient to start with a reuse measurement plan, a FP form and a customizable report template for presenting the results.

- Think out a strategy of how to maximize the benefits of the business engineering tools the team uses in the course of the ERP implementation. These can be of great support to the measurement process.

- Use the data for planning action items.

\section{Conclusions}

This paper has looked in depth at the most important planning aspects of requirements reuse measurement in ERP projects. We blended stakeholder interaction analysis with a process integration model that ensures the visibility of both reuse measurement and RE activities. This resulted in a practical requirements reuse measurement plan that could be applied incrementally to selected portion of the business requirements as well as to the entire project. The plan documents the components of a consistent measurement process: relevant stakeholders, a RE process model, a process integration model, counting rules, tools and reuse data usage tables. The process is 
kept reasonably simple so that team members can concentrate on their requirements elicitation and negotiation activities with functional size and reuse counting and data report generation playing a supporting role. Experiences of practicing the reuse measurement process have been packaged to derive early lesson learnt and critical success factors for an on-going ERP reuse measurement initiative.

It is the believe of the author, that the plan is applicable beyond the ERP RE context of SAP R/3 implementation projects. The reuse measurements work best where there exists reference process and data models of the business application components being implemented. Thus, the approach could find applicability in implementation projects based on other ERP packages, for example BAAN and Peoplesoft. In addition, it can be used in business applications where business process modelling captured the interactions in the business workflow and the data objects from the business area. Next, different company-specific or project-specific aspects, for example project communication [20], process maturity and stability, might lead to shortening or eliminating of some stages in reuse metrics planning. Basically, the decision for how to handle reuse at requirements level is a risk-based one and depends on the assessment of the risk of having the customization of a standard package out of control versus the costs and the residual risk of each possible reuse handling option.

We consider the work reported in this article as only the beginning of an ongoing effort to develop better requirements reuse measurement practices. Further work will take several directions. One is to build a lessons learnt architecture by using product/process dependency models [7]. Second, it would be interesting to investigate some ERP effort estimation models based on our measures. Lastly, we plan to develop an approach to evaluating the added value benefits from ERP requirements reuse.

The author would like to thank the anonymous reviewers whose comments led to the improvement of this paper.

\section{References}

1. Curran, T., A. Ladd, SAP R/3 Business Blueprint, Understanding Enterprise Supply Chain Management, $2^{\text {nd }}$. Edition, Prentice Hall, Upper Saddle River, NJ (1999)

2. Daneva M.: Mesuring Reuse of SAP Requirements: a Model-based Approach, Proc. Of $5^{\text {th }}$ Symposium on Software Reuse, ACM Press, New York (1999)

3. Daneva, M., Deriving Function Points from SAP Business Processes and Business Objects, Journal of Information and Software Technologies (1999). Accepted for publication

4. Daneva M., Empirical Validation of Requirements Reuse Metrics. In preparation.

5. Desharnais, J.-M., A. Abran, How to Successfully Implement a Measurement Program: From Theory to Practice. In: Müllerburg, M., Abran A. (eds.): Metrics in Software Evolution, R. Oldenbourg Verlag, Oldenburg (1995), 11-38.

6. ESPRIT Project PROFES, URL: http://www.ele.vtt.fi/profes.

7. Fenton, N., Pfleeger, S.L.: Software Metrics: Rigorous and Practical Approach, PWS Publishing, Boston Massachusetts (1997)

8. Garmus D., D. Herron, Measuring the Software Process, Prentice Hall, Upper Saddle River, New Jersey (1996). 
9. Jacquet, J.-P., Abran, A.: Metrics Validation Proposals: a Structured Analysis. In: Dumke, R., Abran, A. (eds.): Software Measurement, Gabler, Wiesbaden (1999), 43-60.

10. Karlsson, E.-A. (ed.): Software Reuse, John Wiley \& Sons, Chichester (1998)

11. Keller, G., Teufel, T.: SAP R/3 Process Oriented Implementation, Addison-Wesley Longman, Harlow (1998)

12. Lozinsky, S.: Enterprise-wide Software Solutions, Addison-Wesley, Reading MA (1998)

13. McClure, C.: Reuse Engineering: Adding Reuse to the Software Development Process, Prentice-Hall, Upper Saddle River, NJ (1997)

14. Pfleeger, S.L.: Measuring Reuse: a Cautionary Tale, IEEE Software, June (1997)

15. Poulin, J. Measuring Software Reuse: Principles, Practices, and Economic Models, Addison-Wesley, Reading, MA (1997)

16. Robinson, S. , J. Robinson, Mastering the Requirements Process, Addison- Wesley, Readings, MA (1999)

17. Sharp, H., A. Finkelstein, G. Galal, Shakeholder Identification in the Requirements Engineering Process, Proceeding of the $1^{\text {st }}$ Intl. Workshop on RE Processes/ $10^{\text {th }}$ Intl Conf. on DEXA, 1-3 Sept., 1999, Florence, Italy.

18. Statz, J., Leverage Your Lessons, IEEE Software, March/April (1999), 30-33.

19. Welti, N., Sussessful R/3 Implementation, Practical Management of ERP Projects, AddisonWesley, Harlow, England (1999). 